\title{
CROSSTALK MEASURE OF THE FM - EDFA IN MDM TRANSMISSION USING PM-QPSK
}

T. Senthil

Ph.D., Kalasalingam University. B.E and M.E degree, M.K university, India. Associate Professor, department of ECE, Kalasalingam University,

Krishnankoil, Tamilnadu, (India).

E-mail: t.senthil@klu.ac.in

ORCID: https://orcid.org/0000-0002-0044-5371

\section{Citación sugerida:}

Senthil, T. (2021). Crosstalk measure of the FM - EDFA in MDM Transmission using PM-QPSK. 3C Tecnología. Glosas de innovación aplicadas a la pyme, Edición Especial, (noviembre, 2021), 231-241. https:// doi.org/10.17993/3ctecno.2021.specialissue8.231-241 


\section{ABSTRACT}

In optical fiber communications, Mode -Division Multiplexing in combination with Wavelength Division Multiplexing and advanced modulation formats like QPSK n-QAM are utilized to deliver more amounts of data through a single fiber. For amplifying the signals in the long haul optical fibres link Few -Mode Erbium -Doped Fiber Amplifier is employed to equally and efficiently amplify both modes and respective wavelengths over the telecommunication C-band frequency range. In this paper A Dual-Carrier PM-QPSK transmission system is proposed for enhancing data rate with reduced bit error due to the cross section generated by the optical fibre non-linear properties. The spectrally efficient high bitrate transmission has generated in coherent systems. Using two PM-OPSK carriers, one can reduce transmitter bandwidth requirement to half compared to standard single carrier PM-QPSK while still supporting the same total bitrate. The total bitrate for this layout is given as 40Gbps. The $100-\mathrm{Km}$ of fibre is modelled via a loss element and the fiber dispersion is modelled by an FBG model. Low transmitted power values in coherent systems typically give lower nonlinearity. Each sub-carrier is modulated by $21.4 \mathrm{Gbps}$ including FEC overheads. That means each polarization of a carrier is modulated by $10.7 \mathrm{Gbps}$ implying the symbol rate of $5.35 \mathrm{GHz}$. In order to minimize the crosstalk between the two carrier, the separation between them is kept half the symbol rate. As a result, when one carrier is at maximum, the other is at its minimum. The proposed scheme is utilized for both linear and non-linear fiber.

\section{KEYWORDS}

DC-PM- QPSK, Forward Error Correction, Fibre Bragg Grating. 


\section{INTRODUCTION}

In electronics and telecommunication, modulation is the process of varying one or more properties of a periodic waveform, called the carrier signal, with a modulating signalthat typically contains information. The optical communication is one of the fastest communication methods. Afive-mode erbium doped amplifier is described, and the spectral and modal gain distribution is characterized using PDM-QPSK (Askarov \& Kahn, 2012). However, the combination of polarization division multiplexing (PDM)or in phase/ quadrature phase modulation formats with (WDM) wavelength division multiplexing in single mode fiber leads to the Shannon limit (Ip, 2012). 41.6 Tbit/s C-band signal is transmitted using 12 spatial \& polarization modes over 74.17Km (Genevaux et al., 2016). Multiplexing and De-multiplexing of each spatial mode is needed which causes loss and need several amplifiers, to amplify all the modes at the same time MDM is an economical benefit (Essiambre et al., 2015). General approach to describe and predict the amplifying properties of FM-EDFA - (Few Mode) (Essiambre et al., 2015). The combination of some erbium doped properties with adapted pump has investigated to develop a FM-EDFA model based on the vector modes (Ryf et al., 2012). In this paper we describe the DualCarrier transmission using PM-QPSK modulation and describes the need for PM-QPSK in section II. Then the result analysis and tabulation are shown in section III. This paper concludes in section IV.

\section{MATERIALS AND METHODS}

In this paper the Dual-Carrier transmission is generated using coherent system like PMQPSK. The total bitrate for the layout is $40 \mathrm{Gbps}$. The system consists of three parts first is the transmission part in which pseudo random bit sequence (PRBS) is used as the data transmission which is modulated using the LASER. Then the modulated data is transmitted through the fiber which the second part is, it consists of the combiner and splitter. The fiber used here is EDFA with $100-\mathrm{Km}$. Then at the third part receiver is placed which will measure the crosstalk between the two carrier. The process is subjected to both linear and non-linearities of the fiber. 


\section{A. MODULATION TECHNIQUE}

Modulation is the process of combining the information signal with the carrier signal. Here we use PM-QPSK to achieve the spectrally efficient transmission. Using two PM-QPSK the transmitter bandwidth can be reduced half as compared to single carrier. Each subcarrier is modulated by $21.4 \mathrm{Gbps}$. This will improve the bandwidth efficiency of the transmission system effectively. The block diagram of PM-QPSK is given below (Figure 2).

\section{B. OPTICAL AMPLIFIER}

EDFA is an Erbium Doped Fiber Amplifier which amplifies the signal is also called as the optical amplifier. EDFA is used to avoid the repeaters / regenerators in long distance optical fibre transmission. If we use regeneratorsmeans, there will be high noise introduced by them. To avoid this, we go for EDFA system. However, the cost of this fiber will be high. Even though EDFA amplifies the modulated signal, it needs an external laser pump signal. The EDFA amplifier block is given below (Figure 3).

\section{CROSS TALK MINIMIZATION}

To minimize the crosstalk between the two carriers the separation between them is kept as half the symbol rate that is when one carrier has the maximum symbol rate means other will be at the minimum. The local frequency is set to the transmitted carrier frequency in single PM-QPSK and so the real and imaginary part of the received electromagnetic is a baseband signal. This drastically reduces the possibility of cross talk occurring by introducing orthogonal property in the modulated signal.

\section{RESULTS}

The separation between the two carrier signals is kept as half the symbol rate. The figure shows the orthogonal nature of the signal so that the maximum point of the one signal coincides with the minimum point of another signal thus reducing the crosstalk nature effectively. The dual polarization itself send the two-information signal thus forming one form of multiplexing.The spectrum of the transmitted dual-carrier PM-QPSK is given below. The bandwidth of the carrier signal is determined by the symbol rate of the baseband signal. Thus, by selecting the appropriate Band Pass Filter in the input section the symbol 
rate can be decided for the required isolation in between two signals thus avoiding the cross talk between the signals.

The BER versus for the various symbol rate data from each carrier is analysed and the equalizer is used in the transmitter part to compensate for all transmission anomalies. The data is received with error free is given below. The BER increases with the increased symbol rate and the crosstalk signal strength i.e. overlapped signal spectrum of the two signals also increases with the symbol rate of the baseband signal. The Mode division multiplexed (MDM) system increases the channel capacity imposed by the non - linear properties of the single mode fibre communication system.

In simulation the dual carrier PM - QPSK is designed and then the fibre is set with the optimized non-linear properties so that the effect of the non - linear nature varying the spectral response of the transmitting fibre. The optical signal and spectrum view is given in the Figure 7. The maximum value of the spectrum coincides with the minimum attenuation windows of the single mode fibre. Then the bit error rate range is given in the Figure 8, for both the modulation end. At the receiver side Fibre Bragg Grating (FBG) is used to compensate the bit error rate. The gain value is calculated which is used to minimize the bit error rate due to the dual carrier nature. The range of gain is $6-6.5 \mathrm{db}$ in our simulation generated. By applying non-linearity of the fibre channel, the resultant bit error rate will be approximately nil for the dual carrier system.

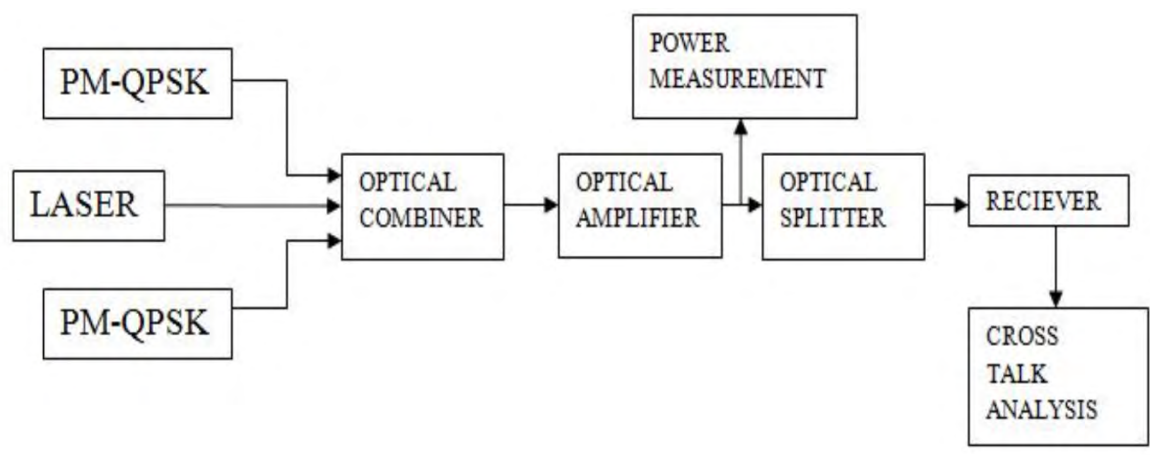

Figure 1. Dual Carrier PM-QPSK system.

Source: own elaboration. 


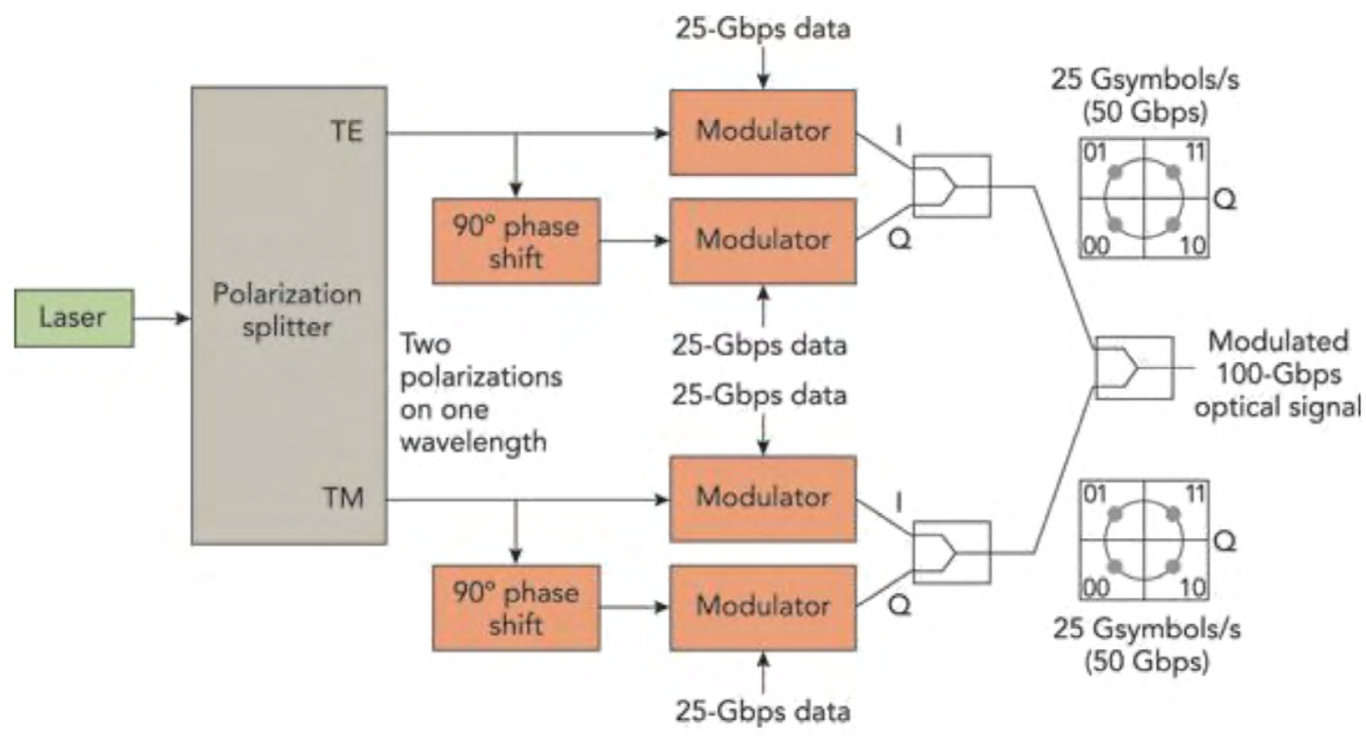

Figure 2. Dual Carrier PM-QPSK concept.

Source: own elaboration.

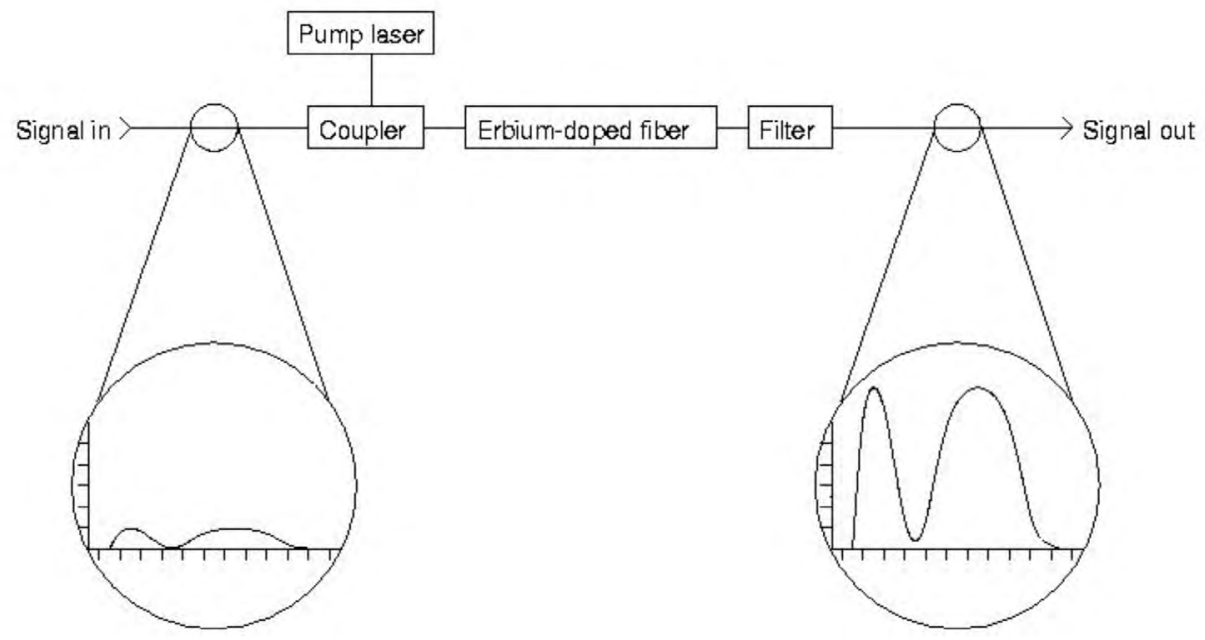

Figure 3. EDFA Working.

Source: own elaboration. 


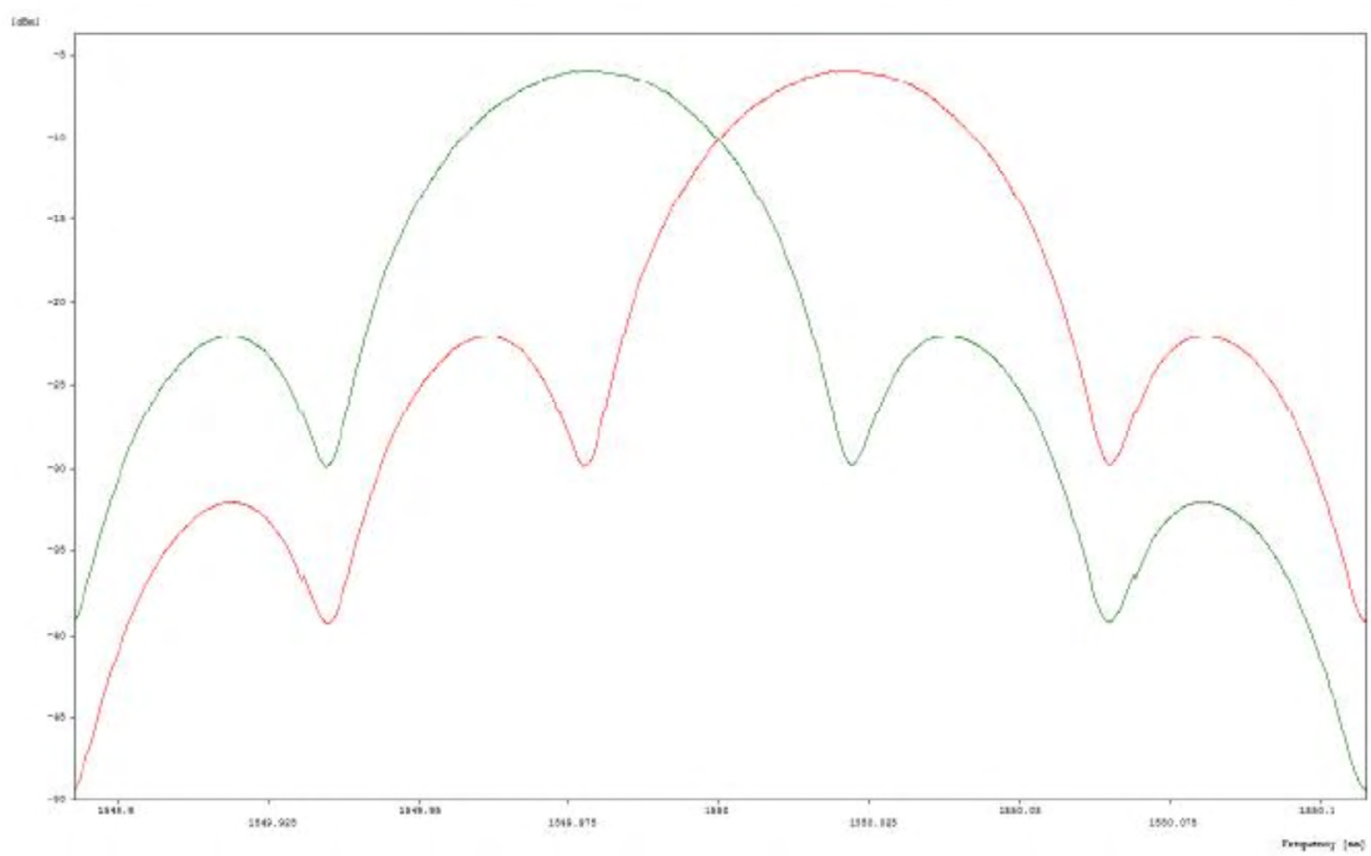

Figure 4. Half the symbol EDFA Working.

Source: own elaboration.

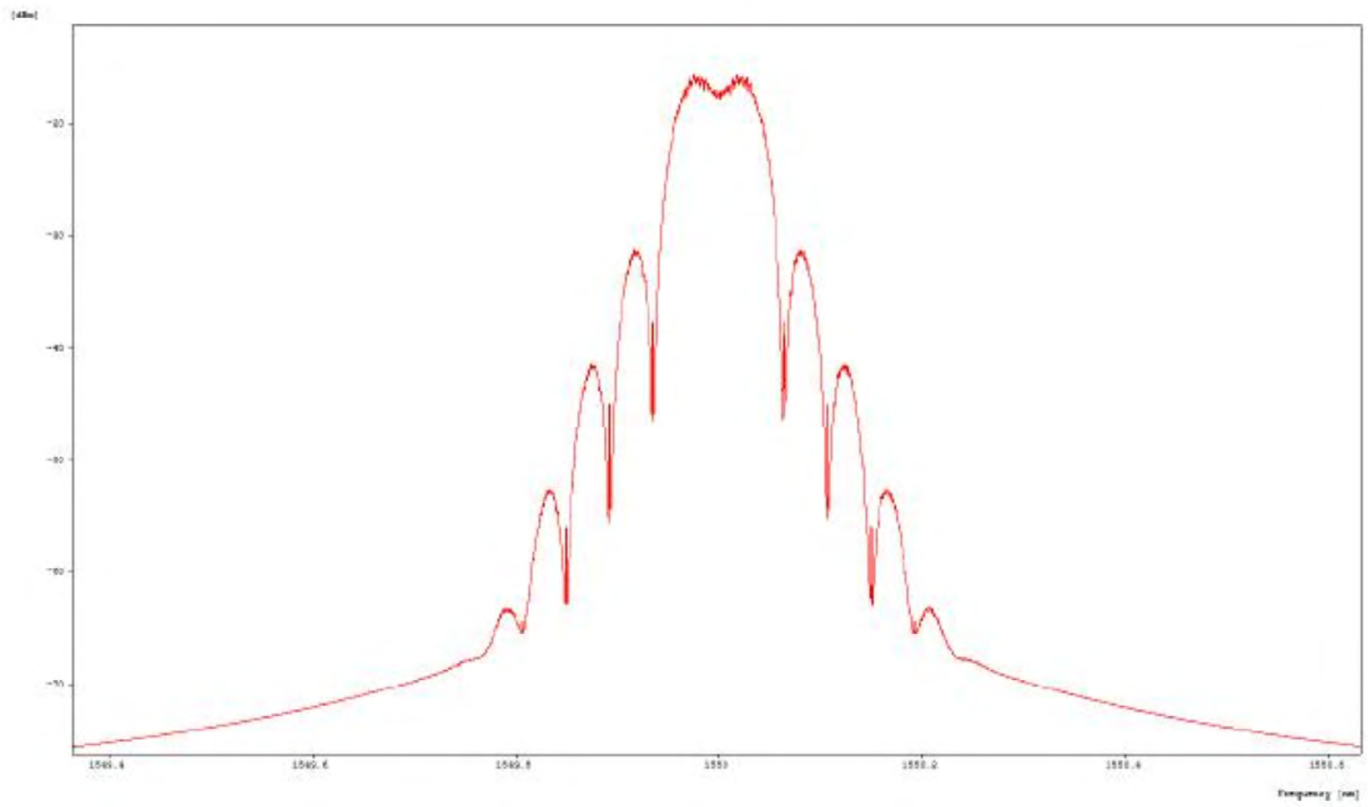

Figure 5. DC-PM-QPSK spectrum at the transmitter.

Source: own elaboration. 

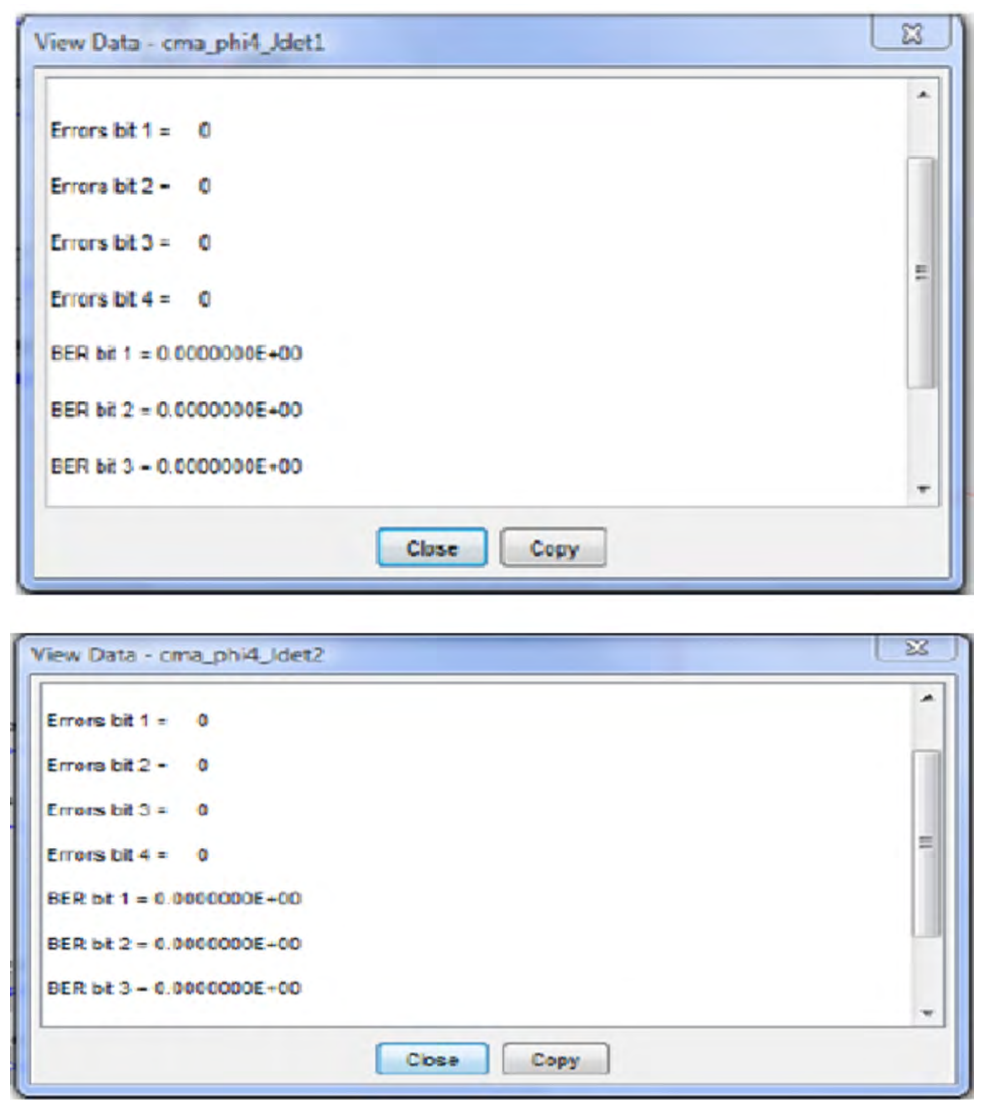

Figure 6. BER for each carrier.

Source: own elaboration.
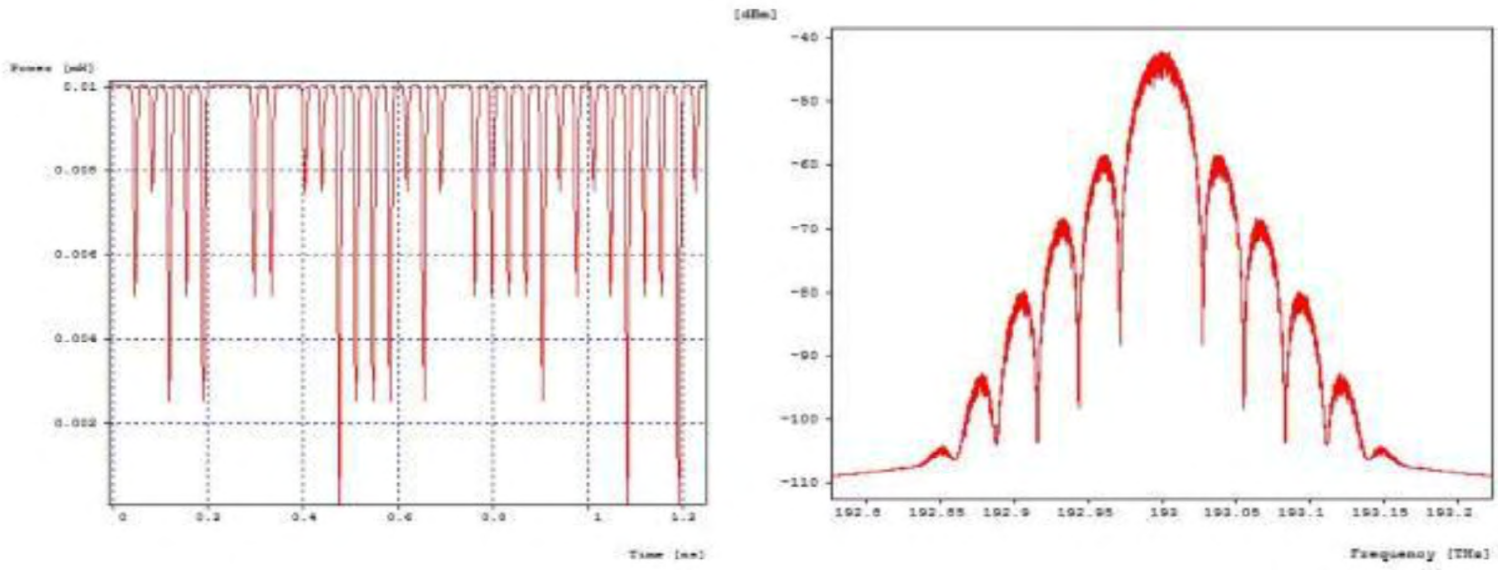

Figure 7. Optical signal and spectrum at the transmitted end.

Source: own elaboration. 


\section{ACKNOWLEDGEMENT}

The author thanks the Department of Electronics and Communication Engineering of Kalasalingam University, (Kalasalingam Academy of Research and Education), Tamil Nadu for permitting, to utilize the OptSim software available in the research lab.

\section{REFERENCES}

Akhtari, S., \& Krummrich, P. M. (2013). Impact of mode beating effects in optical multi-mode amplifiers for space division multiplexing. IEEE Photonics Technology Letters, 25(24), 2482-2485. https://ieeexplore.ieee.org/document/6651699

Askarov, D., \& Kahn, J. M. (2012). Design of transmission fibers and doped fiber amplifiers for mode-division multiplexing. IEEE Photonics Technology Letters, 24(21), 1945-1948. https://ieeexplore.ieee.org/document/6316068

Bigot, L., Le Cocq, G., \& Quiquempois, Y. (2015). Few-mode erbium-doped fiber amplifiers: A review. Fournal of Lightwave Technology, 33(3), 588-596. https://ieeexplore. ieee.org/document/6983523

Chen, Y., Lobato, A., Jung, Y., Ghen, H.,. Sleiffer, V., Kuschnerov, M., Fontaine, N., Ryf, R., Richardson, D., Lank1, B., \& Hanik, N. (2015). 41.6 Tbit/s C-Band SDM OFDM transmission through 12 Spatial and polarization modes over 74.17 Km few mode fiber. Fournal of Lightwave Technology, 33(7), 1440-1444. https://www. osapublishing.org/jlt/abstract.cfm?uri=jlt-33-7-1440

Essiambre, R.-J., Kramer, G., Winzer, P. J., Foschini, G. J., \& Goebel, B. (2015). Capacity limits of optical fiber networks. Fournal of Lightwave Technology, 28(4), 662701. https://ieeexplore.ieee.org/document/5420239

Essiambre, R.-J., Kramer, G., Winzer, P. J., Foschini, G. J., \& Goebel, B. (2015). Capacity limits of optical fiber networks. Fournal of Lightwave Technology, 28(4), 662701. https://ieeexplore.ieee.org/document/5420239

Genevaux, P., Simonneau, G., Le Cocq, G., Quiquempois, Y., Bigot, L., Boutin, A., \& Charlet, G. (2016). A Five Mode Erbium Doped Amplifier For Mode Division 
Multiplexing Transmission. Journal of Lightwave Technology, 34(2), 456-462. https:// ieexplore.ieee.org/document/7274319

Ip, E. (2012). Gain equalization for few-mode fiber amplifiers beyond two propagating mode groups. IEEE Photonics Technology Letters, 24(21), 1933-1936. https: / /ieeexplore. ieee.org/document/6316074

Ryf, R., Randel, S., Gnauck, A. H., Bolle, C., Sierra, A., Mumtaz, S., Esmaeelpour, M., Burrows, E. C., Essiambre, E.-J., Winzer, P. J., Peckham, D. W., McCurdy, A. H., \& Lingle, R. (2012). Mode-division multiplexing over $96 \mathrm{~km}$ of fewmode fiber using coherent $6 \times 6$ MIMO processing. Fournal of Lightwave Technology, 30(4), 521-531. https://ieeexplore.ieee.org/document/6074912

Spellmeyer, N. W. (2000). Communications performance of a multimode EDFA. IEEE Photonics Technology Letters, 12(10), 1337-1339. https://ieeexplore.ieee.org/ document/883822

Wada, M., Sakamoto, T., Mori, T., Yamamoto, T., Hanzawa, N., Yamamoto, N., \& Yamamoto, F. (2014). Modal gain controllable 2-LP-mode fiber amplifier using PLC type coupler and long-period grating. Journal of Lightwave Technology, 32(24), 4694-4700. https://ieeexplore.ieee.org/document/6924757 